\title{
Calculation of Collision Probability Matrix of Nuclear Fuel Cell as a Function of Neutron Energy Group Using Flat Flux Model
}

\author{
Mohammad Ali Shafii \\ Department of Physics, Andalas University, Padang, \\ Indonesia \\ mashafii@sci.unand.ac.id \\ Dian Fitriyani \\ Department of Physics, Andalas University, Padang, Indonesia \\ Seni H J Tongkukut \\ Department of Physics, Sam Ratulangi University, Manado, Indonesia, \\ Zaki Su'ud \\ Nuclear and Biophysics Laboratory, Bandung Institute of Technology, Bandung, Indonesia.
}

Received: January 25, 2021. Revised: August 2, 2021. Accepted: August 24, 2021. Published: August 25, 2021.

\begin{abstract}
One of the methods that widely used in solving neutron transport equations in the nuclear fuel cell is the collision probability (CP) method. The neutron transport is very important to solve because the neutron distribution is related to the reactor power distribution. The important thing in the $C P$ method is the CP matrix calculation, better known as $P_{i j}$ has an important role in determining the neutron flux distribution in the reactor core. This study uses a linear flat flux model in each cell region for each energy group with white boundary condition. Although the type of reactor used in this study is a fast reactor, the $P_{i j}$ matrix calculation still carried out in fast and thermal group energy. The $P_{i j}$ matrix depends on the number of mesh in each cell region. The $P_{i j}$ matrix formed from the mesh distribution will produce a $6 \times 6$ matrix for each energy group. Because the boundary condition of the system is assumed that there are no contributions neutron source from the outside, the
\end{abstract}

sum of the $P_{i j}$ matrix must be less than one. In general, the results of the calculations $P_{i j}$ in this study are following the theory.

Keywords-A CP method, $P_{i j}$ matrix, energy group, nuclear fuel cell, linear flat flux model

\section{INTRODUCTION}

$\mathrm{T}$ HE calculation of neutron transport and its interaction with matter is a fundamental topic in reactor physics [1]. The most complicated part of the nuclear reactor is to solve the integral neutron transport equation as a function of energy, space, and time. Neutron transport investigates to determine the distribution of neutrons in the reactor core, that is, the motion of the neutrons, the scattering process when its interaction with nuclei and the process of a neutron being absorbed or leaking out of the reactor [2]. One of the methods that widely used in solving neutron transport equations in the 
nuclear fuel cell is the collision probability (CP) method. Solving an integral transport equation with the $\mathrm{CP}$ method generally uses the linear flat flux model; namely, the neutron flux in each volume region is considered constant [3],[4]. This model allows neutron flux to be maximal in reactor fuel because neutrons move in all directions and will decrease when it reaches shielding limits. The linear flat flux model often used in the CP method to solve neutron transport problems in nuclear fuel cells [5]. CP method has an advantage for simple and symmetrical geometry such as a cylindrical cell.

The review of the flux points in each region is necessary to demonstrate the neutron flux distribution in each region in the nuclear fuel cell. Flux points with certain intervals in the fuel cell are called mesh points. If the distance between the mesh is the same, then the neutron flux in each mesh is considered a flat flux model. Because the total cross-section in each region is considered constant, the neutron flux and neutron source in the region can be considered constant as well. The calculation of the neutron flux distribution cannot be executed directly, because in general, the spatial mesh order is very small; neither is the energy range. Thus, the amount of total mesh that must be done for the entire core becomes very large. For this reason, fuel cell homogenization is done by the CP method [6].

The important thing in the $\mathrm{CP}$ method is the $\mathrm{CP}$ matrix calculation or better known as $P_{i j}$ matrix. Handling the geometry of nuclear fuel cells is usually done by limiting the choice of cell geometry to be calculated. Furthermore, for each type of geometry, CP matrix calculations are performed by using analytic integration on angles, as well as numerical calculations for complex cases, such as the calculation of the Bickley-Naylor function, and the Dancoff factor [7]. Thus, if a certain computer code does not have the right geometry choice, to add geometric capabilities to the computer code is a very great effort.

In the previous study [8], the Calculation of $P_{i j}$ matrix in the one-dimensional slab geometry of nuclear fuel cells using the $\mathrm{CP}$ method has been carried out. This study uses the CP method is implemented on the one-dimensional cylindrical nuclear fuel cell with the FF approach as a function of 70 energy groups for fast reactor type. The $P_{i j}$ matrix calculated in this study is the $P_{i j}$ matrix for scattering and escape processes. The library data used is JFS-3-J33 from JAEA Japan. The $P_{i j}$ matrix calculation results obtained in this study will then be used in the calculation of neutron flux distribution in the further studies.

\section{THEORETICAL BACKGROUND}

The neutron probability that is emitted thoroughly and isotropic in the $\mathrm{i}$ region and subsequently collides in $\mathrm{j}$ region within the cylindrical nuclear fuel cell for each energy group $g$ is following [9]

$$
\Sigma_{j g} V_{j} \phi_{j g}=\frac{1}{k_{e f f}} \sum_{i} V_{i} P_{i j g} S_{i g}
$$

Where $V_{i}=\pi\left(r_{i}^{2}-r_{i-1}^{2}\right)$ and $\Sigma_{j g}$ is a macroscopic crosssection total in the $j$ region. The CP matrix in equation (1) is [10]

$$
\begin{aligned}
P_{i j}=\frac{2}{\sum_{i} V_{i}} \int_{0}^{r_{i}} d \rho\left[K_{i 3}\left(\lambda_{i j}^{1}\right)-K_{i 3}\left(\lambda_{i j}^{1}+\lambda_{i}\right)-K_{i 3}\left(\lambda_{i j}^{1}+\lambda_{j}\right)+K_{i 3}\left(\lambda_{i j}^{1}+\lambda_{i}+\lambda_{j}\right)\right. \\
\left.+K_{i 3}\left(\lambda_{i j}^{2}\right)-K_{i 3}\left(\lambda_{i j}^{2}+\lambda_{i}\right)-K_{i 3}\left(\lambda_{i j}^{2}+\lambda_{j}\right)+K_{i 3}\left(\lambda_{i j}^{2}+\lambda_{i}+\lambda_{j}\right)\right]
\end{aligned}
$$

Where

$$
\lambda_{k}=\Sigma_{k}\left(x_{k}-x_{k-1}\right), \lambda_{i j}^{2}=\sum_{k=1}^{i-1} \lambda_{k}+\sum_{k=1}^{j-1} \lambda_{k} \text { and } \lambda_{i j}^{1}=\sum_{k=i-1}^{j-1} \lambda_{k}
$$

The $K_{i j}(\lambda)$ is the third order of the Bickley-Nayler function. For cases of CP matrix that occur on the $i$ region the same as in the $j$ region (self-collision probability), the CP matrix in equation (2) becomes to [10] 


$$
\begin{array}{r}
P_{i i}=\frac{2}{\sum_{i} V_{i}} \int_{0}^{r_{i-1}} d \rho\left[2 \lambda_{i}-2 K_{i 3}(0)+2 K_{i 3}\left(\lambda_{i}\right)+K_{i 3}\left(\lambda_{i i}\right)-2 K_{i 3}\left(\lambda_{i i}+\lambda_{i}\right)\right. \\
\left.+K_{i 3}\left(\lambda_{i i}+2 \lambda_{i}\right)\right]+\frac{2}{\sum_{i} V_{i}} \int_{r_{i-1}}^{r_{i}} d \rho\left[2 \lambda_{i}-K_{i 3}(0)+K_{i 3}\left(2 \lambda_{i}\right)\right]
\end{array}
$$

The boundary condition used in this study is white boundary conditions which means a reflective condition where all neutron striking the boundary turn back with an isotropic angular distribution. The white boundary condition is usually explained as an isotropic reflection. The computational results are more accurate and time-consuming of the computational process less compared to other boundary conditions. It is assumed that there are no contributions of neutron source from outside. The CP matrix will change because neutrons emitted in the $i$ region will come out of the outer boundary without experiencing any collision (escape probability), so the $\mathrm{CP}$ matrix in equation (3) becomes to [10]

$P_{i s}=\frac{2}{\sum_{i} V_{i}} \int_{0}^{r_{i}} d \rho\left\{K_{i 3}\left(\lambda_{i s}^{1}\right)-K_{i 3}\left(\lambda_{i s}^{1}+\lambda_{i}\right)+K_{i 3}\left(\lambda_{i s}^{2}\right)-K_{i 3}\left(\lambda_{i s}^{2}+\lambda_{i}\right)\right\}$

Where

$$
\lambda_{i s}^{1}=\sum_{k=i+1}^{N} \lambda_{k} \text { and } \lambda_{i s}^{2}=\sum_{k=1}^{i-1} \lambda_{k}+\sum_{k=1}^{N} \lambda_{k} .
$$

\section{NuClear FuEl Design AND COMPUTATIONAL} METHOD

This study only focuses on the completion of the neutron transport process in a nuclear fuel cell. Selected cell geometry is cylindrical, divided into three regions, namely: region 1 is fuel, region 2 is cladding, and region 3 is coolant [11]. Table 1 shows the specification design of the nuclear fuel cell. The nuclear fuel used in this study is a mixture of U-Pu Nitride; the cladding structure material is stainless steel and $\mathrm{Pb}-\mathrm{Bi}$ as a coolant. Energy group data were selected for 70 groups based on the energy group structure SLAROM JFS-3-J33 [12].

Table 1. Specification design of nuclear fuel cell [6].

\begin{tabular}{ll}
\hline \multicolumn{1}{c}{ Parameters } & \multicolumn{1}{c}{ Specification } \\
\hline Type of nuclear fuel & Uranium-Plutonium \\
cell & Nitride \\
Geometry of cell & Cylindrical 1D \\
Material Structure & Stainless steel \\
Coolant & Lead-Bismuth (Pb-Bi) \\
Diameter of pin cell & $1.134 \mathrm{~cm}$ \\
Cladding thickness & $0.11 \mathrm{~cm}$ \\
Temperature Average & $1183 \mathrm{~K}$ \\
Volume fraction: fuel & $61.73 \%$ \\
structure & $19.40 \%$ \\
coolant & $18.87 \%$ \\
\hline
\end{tabular}

The outline of the $\mathrm{CP}$ matrix calculation program and its computational aspects are as follows [13]-[15]: After reading the total macroscopic cross-section value calculated from the homogenizing nuclear fuel cells process based on library data. The volume of the cell for each region is determined to run equation (1). The scattering cross-section, neutron fission production, absorption cross-section are calculated for each volume region and energy group. The $\mathrm{CP}$ matrix is calculated for all regions divided into amount mesh, and the energy group uses equation (2) and (3). The probability escape matrix is calculated by completing the equation (4). Iteration is done to recalculate the $\mathrm{CP}$ matrix and escape probability based on equation (1) to convergence. The computational program has been written in Delphi. The iteration is executed for six meshes and 70 groups of energy.

\section{RESULTS AND DISCUSSION}

The calculation of $P_{i j}$ the matrix in this study uses the FF approach in each region on all energy group. When the scattering neutron cross-section is homogeneous in the entire region, the $P_{i j}$ matrix remains constant for the entire i region.

This condition occurs because the neutron flux distribution becomes flat evenly distributed in all cell regions. In most heterogeneity systems, spatial flux is considered to be flat, especially outside resonance. This $P_{i j}$ matrix depends on the number of mesh in each cell region. The matrix $P_{i j}$ formed from the mesh distribution will produce an $n \times n$ matrix, with $n$ is the number of mesh in each region. For example, if the total mesh in the cell is six meshes, so the formed $P_{i j}$ matrix is a $6 \times 6$ matrix for each energy group. If the total mesh is raised to 10 meshes, the $P_{i j}$ matrix will form a 10x10 matrix and so on. The calculation results of the $P_{i j}$ matrix for the first three energy groups of fast energy that represent high energy are shown in Figure 1 and the last three energy groups representing thermal energy can be seen in Figure 2 . The escape probability matrix will form a $1 \times n$ matrix because in this event neutrons born in the $i$ region do not experience collisions in the $j$ region. The $\mathrm{CP}$ matrix produced using the $\mathrm{FF}$ approach is used to calculate the neutron flux using equation (1).

In the SLAROM library, the division of the JFS-3-J33-70 group energy group structure for high energy ( $\mathrm{MeV}$ order) is represented by group 1 to group 19, medium energy ( $\mathrm{keV}$ order) represented by group 20 to group 37, while low energy ( $\mathrm{eV}$ order) is represented by groups 38 to group 70. In general, the division of the energy group is divided into two regions, 
namely the fast region, which is represented by groups 1 to 37 and thermal regions represented by groups 38 to 70 [12].

From Figures 1 and 2, it can be concluded that in the cylinder geometry, the calculation results of the $P_{i j}$ matrix are very close in a region with the same energy, both in high energy region and in low energy region. Moreover, the matrix values $\mathrm{P} 11, \mathrm{P} 12$, and $\mathrm{P} 13$ are greater than $\mathrm{P} 14, \mathrm{P} 15$, and $\mathrm{P} 16$, because the composition of nuclear fuels are in the regions 1 to
3 where, in this regions, there will be a lot of nuclear fission processes, while in the region 4 to 6 that contain cladding and coolant do not occur nuclear fission processes. In addition, the macroscopic cross-section of the total reaction in the fuel region, is too large compared to the macroscopic cross-section of the total reaction in the cladding and coolant.

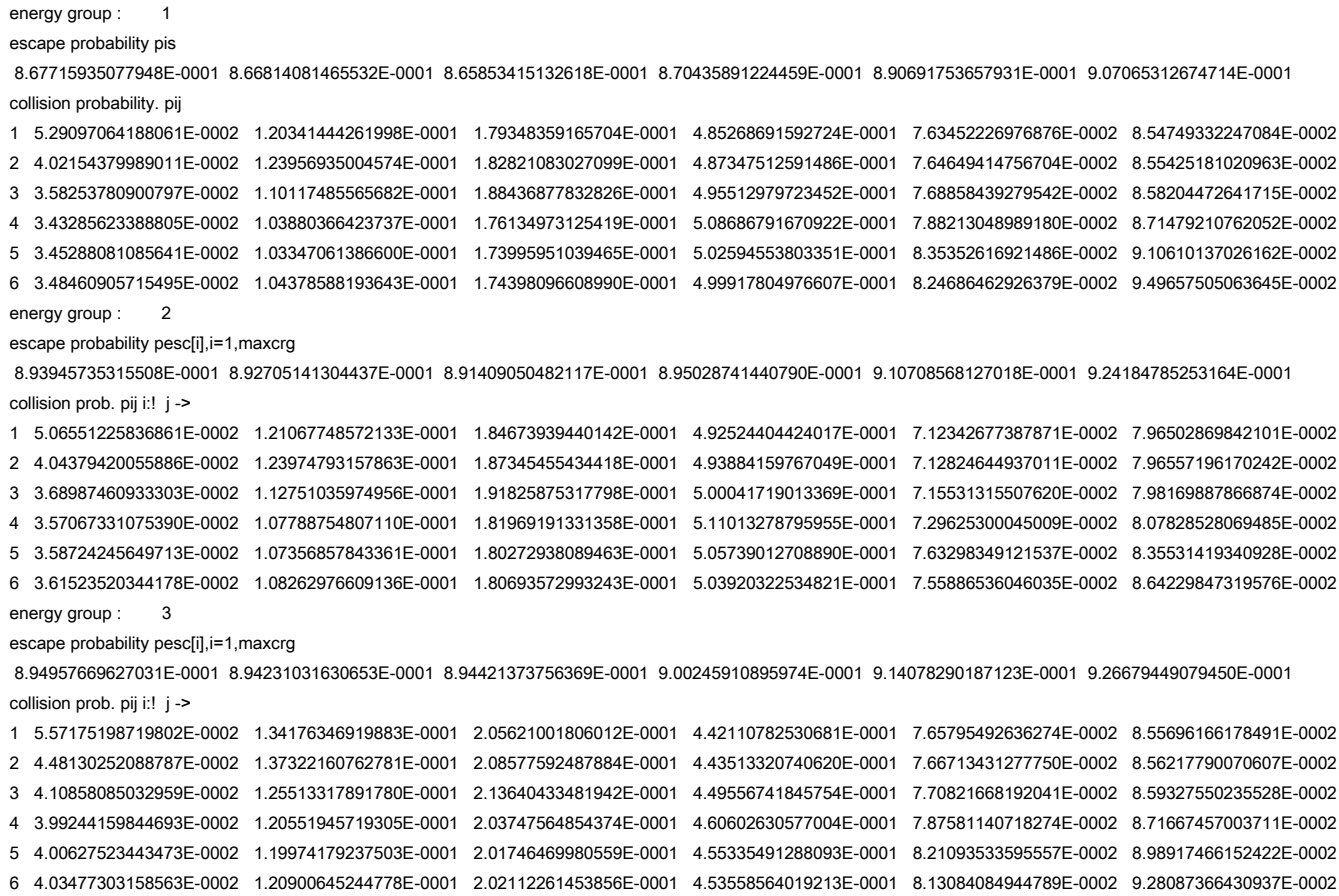

Fig. 1 The $P_{i j}$ matrix for the first three energy groups of fast energy.

energy group : $\quad 68$
escape probability pesc[i],i=1, maxcrg

4.67808165638431E-0001 4.97377902076859E-0001 5.75878866768582E-0001 7.04410505169733E-0001 7.58217699406339E-0001 7.92008809477216E-0001 collision prob. pij i:! j ->

$12.00025855529713 E-0001 \quad 2.92471647400913 E-0001 \quad 3.36158655392559 E-0001 \quad 8.67794820878681 E-0002 \quad 3.11051367101737 E-0002 \quad 3.50373630644330 E-0002$ $\begin{array}{llllllll}2 & 9.82147584312396 \mathrm{E}-0002 & 3.14612002078689 \mathrm{E}-0001 & 3.85675516324146 \mathrm{E}-0001 & 9.38526240368571 \mathrm{E}-0002 & 3.33856965274228 \mathrm{E}-0002 & 3.75127619641561 \mathrm{E}-0002\end{array}$

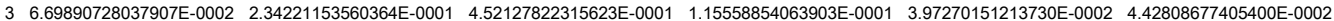
$\begin{array}{lllllll}4 & 6.58650874374331 \mathrm{E}-0002 & 2.16042254942740 \mathrm{E}-0001 & 4.44717593845557 \mathrm{E}-0001 & 1.53905791204464 \mathrm{E}-0001 & 5.23835288362192 \mathrm{E}-0002 & 5.64649155111595 \mathrm{E}-0002\end{array}$ $\begin{array}{lllllll}5 & 6.73881188343796 \mathrm{E}-0002 & 2.14856850962092 \mathrm{E}-0001 & 4.31309085236655 \mathrm{E}-0001 & 1.48700097573067 \mathrm{E}-0001 & 6.19276201946634 \mathrm{E}-0002 & 6.44996763062556 \mathrm{E}-0002\end{array}$ $\begin{array}{llllllll}6 & 6.84703486721841 \mathrm{E}-0002 & 2.18674255115558 \mathrm{E}-0001 & 4.28819084741763 \mathrm{E}-0001 & 1.44025027544208 \mathrm{E}-0001 & 5.89965031294328 \mathrm{E}-0002 & 7.15827344177218 \mathrm{E}-0002\end{array}$ energy group : 69 
Fig. 2 The $P_{i j}$ matrix for the last three energy groups of th ermal energy.

Because the boundary condition of the system is assumed that there are no contributions from outside, the consequence of this boundary condition is the sum of the $P_{i j}$ matrix must be less than one $\left(\sum_{j} P_{i j}<1\right)$ for all mesh due to leakages from the system as shown in Figures 1 and 2. In other words, the results of the CP matrix calculation are following the reference [16][18]. The results of this $P_{i j}$ matrix are used as the basis for nuclear fuel cell design to estimate the shell temperature of fuel elements in former nuclear fuel storage facilities for the needs of reactor construction and safe operation [19]-[20].

\section{CONCLUSIONS}

The CP method, which is based on integral transport, has proven to be very effective and suitable in solving the problem of neutron transport in nuclear reactors, especially for calculating the CP matrix in each region in nuclear fuel cells. The description of the CP matrix is very close in a region with the same energy, both in high energy regions and in low energy region. Because the boundary condition of the system is assumed that there are no contributions from outside, the sum of the $P_{i j}$ matrix must be less than one that is following the reference. The $\mathrm{CP}$ matrix calculation has an important role in determining the neutron flux distribution in the reactor core.

\section{ACKNOWLEDGMENTS}

This research is funded by the Directorate of Research and Community Service Directorate General of Strengthening Research and Development Ministry of Research, Technology, and Higher Education Republik Indonesia under the Research Contract Number: 63/ SP2H/ AMD/ LT/DRPM/2020 for the Fundamental Research scheme.

\section{References}

[1] S. Miwa, Y. Yamamoto, G. Chiba. Research activities on nuclear reactor physics and thermal-hydraulics in Japan after Fukushima-Daiichi accident. Journal of Nuclear Science and Technology, 2018, Vol. 55, No. 6, pp. 575598.

[2] E. Cervi, A. Cammi, E. Zio. A new approach for nuclear reactor analysis based on complex network theory. Progress in Nuclear Energy, 2019, Vol. 112, pp. 96-106.

[3] M. Lahdour, T. El Bardouni, E. Chakir, K. Benaalilou, M. Mohammed, H. Bougueniz, H. El Yaakoubi, H. NTPERSN: a new package for solving the multigroup neutron transport equation in a slab geometry. Applied Radiation and Isotopes, 2019, Vol. 145, pp. 73-84.

[4] T. Younkin, D. L. Green, A. B. Simpson, B. D. Wirth, B. D. GITR: An accelerated global scale particle tracking code for wall material erosion and redistribution in fusion relevant plasma-material interactions. Computer Physics Communications, 2021, Vol. 264, pp. 107885.

[5] S. Choi, D. Lee. Three-dimensional method of characteristics/diamond-difference transport analysis method in STREAM for whole-core neutron transport calculation. Computer Physics Communications, 2021, Vol. 260, pp. 107332.

[6] D. Litskevich, B. Merk. SP3 solution versus diffusion solution in pin-by-pin calculations and conclusions concerning advanced methods. Journal of Computational and Theoretical Transport, 2014, Vol. 43, No. 1-7, pp. 214-239.

[7] M. A. Shafii, Analysis of Neutron Fission Reaction Rate in the Nuclear Fuel Cell Using Collision Probability Method with Non Flat Flux Approach. In Journal of Physics: Conference Series, 2017, Vol. 877, No. 1, pp. 012013

[8] M. A. Shafii, J. Usman, S. H. Tongkukut, A. G. Abdullah. The Pij matrix and flux calculation of one-dimensional neutron transport in the slab geometry of nuclear fuel cell using collision probability method. In MATEC Web of Conferences 2018, Vol. 197, pp. 02006

[9] Z. Suud, M. A. Shafii, R. S. R. SNM. Fission Yield Calculation Method and its Effect in Nuclear Fuel Cell Homogenization Calculation. Indonesian Journal of Physics, 2009, Vol 20, No. 2, pp. 33-36.

[10] K. Okumura, T. Kugo, K. Kaneko, K. Thuchihashi, K. A comprehensive neutronics calculation code system. Japan, JAEA, 2006, pp. 4-26.

[11]H. A. Yousef, A. H. El-Farrash, A. A. Ela, Q. Merza. Measurement of radon exhalation rate in some building materials using nuclear track detectors. World Journal of Nuclear Science and Technology, 2015, Vol. 5, No 03, pp. 141.

[12] T. Hazama, G. Chiba, K. Sugino. Development of a fine and ultra-fine group cell calculation code SLAROM-UF for fast reactor analyses. Journal of nuclear science and technology, 2006, Vol. 43, No. 8, pp. 908-918.

[13] O. Safarzadeh, A. Minuchehr, A. S. Shirani, Heterogeneous reactor core transport technique using response matrix and collision probability methods. Annals of Nuclear Energy, 2013, Vol. 62, pp. 137-143.

[14] C. Zhen-Ping, Z. Hua-Qing, S. Guang-Yao, S. Jing, H. LiJuan, H. Li-Qin, W. Yi-Can, Preliminary study on CADbased method of characteristics for neutron transport calculation. Chinese Physics C, 2014, Vol. 38, No. 5, art. id. 058201 .

[15] S. Ray, S. B. Degweker, R. Rai, K. P. Singh, A collision probability and MOC-based lattice and burnup code for analysis of LWR fuel assemblies. Nuclear Science and Engineering, 2016, Vol. 184, No. 4, pp. 473-494.

[16]D. Altiparmakov, R. Wiersma, The collision probability method in today's computer environment. Nuclear Science and Engineering, 2016, Vol. 182, No. 4, pp. 395416. 
[17]D. Litskevich, B. Merk, S. Atkinson, Verification of the current coupling collision probability method with orthogonal flux expansion for the case of single cell. Progress in Nuclear Energy, 2020, Vol. 120, art. id. 103219.

[18] T. Boševski, An Improved Collision Probability Method for Thermal-Neutron-Flux Calculation in a Cylindrical Reactor Cell. Nuclear Science and Engineering, 1970, Vol. 42, No. 1, pp. 23-27.

[19] I. V. Kazachkov, M. Yu. Kamaev, V. M. Khutornyi R. Ya. Tomyak, Modeling of the Thermal Processes in Repository of the Waste Nuclear Fuel with Water for Assessment of Potentially Hazardous Situations, WSEAS Transactions on Heat and Mass Transfer, 2019, Vol. 14, No. 17, pp. 137-146.

[20]T. Watanabe, Numerical Simulation of Droplet Combustion using Volume-of-Fluid Method, WSEAS Transactions on Heat and Mass Transfer, 2019, Vol. 14, No. 4, pp. 38-44.
Creative Commons Attribution License 4.0 (Attribution 4.0 International, CC BY 4.0)

This article is published under the terms of the Creative Commons Attribution License 4.0

https://creativecommons.org/licenses/by/4.0/deed.en_US 